\title{
Strong Wet and Dry Adhesion by Cupped Microstructures
}

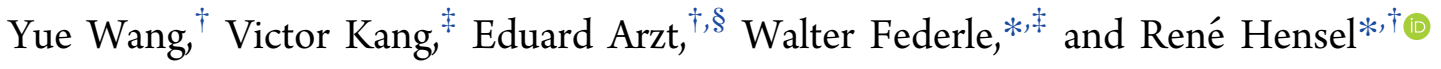 \\ ${ }^{\dagger}$ INM - Leibniz Institute for New Materials, Campus D2 2, Saarbrücken 66123, Germany \\ ${ }^{\ddagger}$ Department of Zoology, University of Cambridge, Cambridge, United Kingdom \\ ${ }^{\S}$ Department of Materials Science and Engineering, Saarland University, Campus D2 2, Saarbrücken 66123, Germany
}

\section{Supporting Information}

ABSTRACT: Recent advances in bio-inspired microfibrillar adhesives have resulted in technologies that allow reliable attachment to a variety of surfaces. Because capillary and van der Waals forces are considerably weakened underwater, fibrillar adhesives are however far less effective in wet environments. Although various strategies have been proposed to achieve strong reversible underwater adhesion, strong adhesives that work both in air and underwater without additional surface treatments have yet to be developed. In this study, we report a novel design-cupped microstructures (CM) - that generates strong controllable adhesion in air and underwater. We measured the adhesive performance of cupped polyurethane microstructures with three different cup angles $\left(15,30\right.$, and $\left.45^{\circ}\right)$ and the same cup diameter of $100 \mu \mathrm{m}$ in dry and wet conditions in comparison to standard mushroomshaped microstructures (MSMs) of the same dimensions. In air, $15^{\circ} \mathrm{CM}$ performed comparably to the flat MSM of the same size with an adhesion strength (force per real contact area) of up to $1.3 \mathrm{MPa}$, but underwater, $15^{\circ} \mathrm{CM}$ achieved 20 times stronger adhesion than MSM ( $\sim 1 \mathrm{MPa}$ versus $\sim 0.05 \mathrm{MPa})$. Furthermore, the cupped microstructures exhibit self-sealing properties, whereby stronger pulls lead to longer stable attachment and much higher adhesion through the formation of a better seal.

KEYWORDS: wet adhesion, self-sealing, cupped microstructures, two-photon lithography, polyurethane

\section{INTRODUCTION}

Over the past decades, fibrillar adhesives inspired by geckos and beetles have shown great potential in numerous applications such as climbing robots, ${ }^{1,2}$ smart manipulators, and grippers ${ }^{3-7}$ because of their strong yet switchable and residue-free adhesive characteristics. $^{8,9}$ In many reports, it was found that the mechanics and design of the tip terminating the fibrils are important in influencing adhesive performance. ${ }^{10-13}$ Among the tip designs, mushroom-shaped ends have been shown to enhance the adhesion force by over 5-10 times compared to simple flat tips by reducing the stress singularity near the cap edge. ${ }^{14-16}$ Yet, when mushroom-shaped microstructures are fully immersed in water, their remarkable adhesion is drastically weakened, ${ }^{17-19}$ especially when the fabrication materials and/or the substrate are hydrophilic. ${ }^{20}$ Investigating the detachment behavior of mushroom-shaped tips, Heepe et al. ${ }^{18}$ proposed that a thin water layer in the contact interface led to lower pull-off forces because of the lower Hamaker constant in water. ${ }^{19,21}$ Other studies also found that interfacial energy and surface wettability play a significant role in the weak underwater adhesion of fibrillar adhesives when tested against a hydrophilic surface. ${ }^{17,20,22}$

Several strategies have been proposed to improve underwater adhesion of fibrillar structures, such as using a chemical adhesive coating on fibrillar surfaces. ${ }^{23-25}$ Lee et al. ${ }^{23}$ demonstrated that mussel-mimetic adhesive proteins applied as thin bioadhesive layers on fibrillar adhesives significantly improved the underwater adhesive performance. Rao et al. ${ }^{26}$ combined nanoscale dynamic bonds in tough hydrogels with macroscale fibrillar designs to achieve strong underwater adhesion. However, thin chemical layers grafted onto fibrillar structures may not be durable for stable and long-term use and could leave chemical residues on the adhered surfaces, while hydrogels are limited to short-term usage in dry conditions.

Ideally, adhesives should perform well in air and underwater without requiring further chemical surface treatments, especially for real-world applications where contact surfaces rapidly alternate between wet and dry from humidity and rain. In the search for solutions, inspiration can be obtained from natural underwater adhesive systems. For example, Baik et al. ${ }^{27}$ demonstrated that dome-shaped protuberances, as found on the surface of octopus suction cups, can increase the adhesion of synthetic microcups on wet surfaces. Their study illustrated that underwater adhesion can be improved by introducing geometric designs without any additional surface treatments.

Received: May 7, 2019

Accepted: June 26, 2019

Published: June 26, 2019 


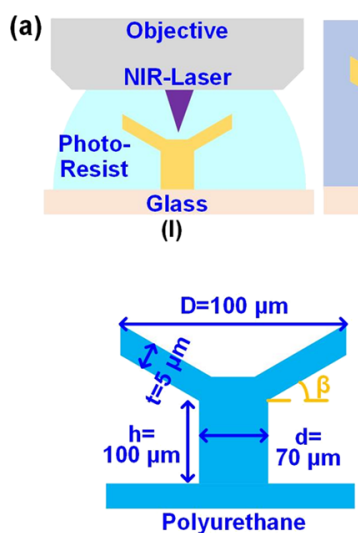

(V)

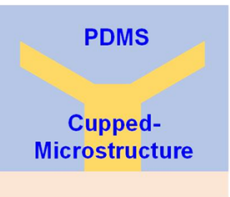

(II)

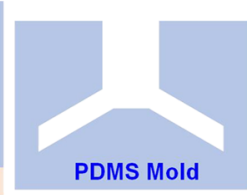

(III)

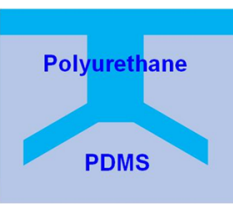

(IV)

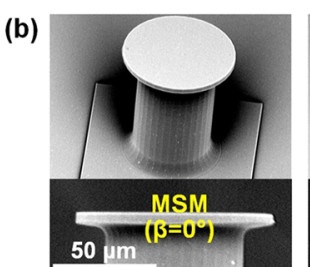

(I)

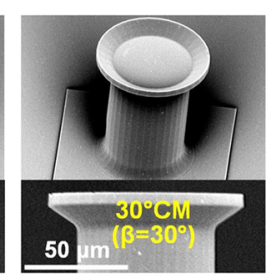

(II)

Figure 1. Fabrication of microstructures with different cap geometries. (a) Illustration of the manufacturing process by (I) two-photon lithography and (II-IV) replica molding. (V) Dimensions of the microstructures. (b) Scanning electron micrographs of fabricated microstructures: (I) mushroomshaped microstructure (MSM) with a flat end $\left(\beta=0^{\circ}\right)$; (II) cupped microstructure $\left(30^{\circ} \mathrm{CM}\right)$ with $\beta=30^{\circ}$ walls. Scale bars: $50 \mu \mathrm{m}$.

Here, to address the challenge of creating adhesives with strong dry and wet adhesion, we have investigated the adhesive performance of novel "cupped" microstructures. By varying the microcup angle (angle between the horizontal and the cup walls from 15 to 30 and $45^{\circ}$ ), we demonstrate that this design allows the production of novel adhesives with excellent performance both in air and underwater.

\section{MATERIALS AND METHODS}

2.1. Microfabrication. Microstructures with a cap diameter of 100 $\mu \mathrm{m}$, a stalk diameter of $70 \mu \mathrm{m}$, and a stalk height of $100 \mu \mathrm{m}$ were designed using Inventor (Autodesk, San Rafael, CA, USA). The threedimensional structures were printed via a two-photon lithography system (Photonic Professional GT, Nanoscribe, Eggenstein-Leopoldshafen, Germany). For printing in dip-in mode, the resin IP-DIP (Nanoscribe, Eggenstein-Leopoldshafen, Germany) was used. Structures were developed by immersion into propylene glycol monomethyl ether acetate (PGMEA, Sigma-Aldrich, St. Louis, MO, USA) for $10 \mathrm{~min}$ and rinsed in isopropanol for $1 \mathrm{~min}$. Before drying with nitrogen, the structures were post-cured by exposing to UV light $(200 \mathrm{~mW}, 365 \mathrm{~nm}$, OmniCure S1500A, Germany) for $5 \mathrm{~min}$ to enhance mechanical stability. ${ }^{28}$ Finally, structures were coated with $(1 \mathrm{H}, 1 \mathrm{H}, 2 \mathrm{H}, 2 \mathrm{H}$ perfluorooctyl)-trichlorosilane (AB111444, ABCR, Karlsruhe, Germany) by a vapor deposition method for $45 \mathrm{~min}$. The structures obtained were used as masters for replica molding (Figure 1a). For this purpose, two-component polydimethylsiloxane (PDMS, Sylgard 184, Dow Corning, Midland, USA) was mixed (10:1 base to catalyst ratio), poured onto master structures, and cured in an oven at $75^{\circ} \mathrm{C}$ for at least $3 \mathrm{~h}$. After demolding, the PDMS template was post-cured on a hot plate at $200{ }^{\circ} \mathrm{C}$ for $30 \mathrm{~min}$. PDMS templates were used (without further surface treatments) for fabrication of polyurethane replicas (PU, NEUKADUR A75, Altropol GmbH, Stockelsdorf, Germany). The PU prepolymer was mixed 1.2 parts base to 1 part cross-linker. To increase the contrast for in situ observation of the adhesion tests, the prepolymer mixture was dyed with 1 wt \% blue pigment (Altropol GmbH, Stockelsdorf, Germany). The dyed PU prepolymer was cast onto the PDMS template and cured in an oven at $65{ }^{\circ} \mathrm{C}$ for at least $3 \mathrm{~h}$. Upon demolding, the polyurethane microstructures were post-cured at 120 ${ }^{\circ} \mathrm{C}$ on a hot plate.

2.2. Adhesion Measurements. Adhesion tests were performed using a custom-made apparatus as shown in Figure S1 in the Supporting Information. It consists of a motorized stage with a minimum step size of $6 \mathrm{~nm}$ (Q-545.240, PI, Karlsruhe, Germany), two goniometers, a sample holder, and a load cell with a resolution of $0.4 \mathrm{mN}(\mathrm{KD} 45-2 \mathrm{~N}$, ME-Messsysteme, Henningsdorf, Germany). The flat face of a glass cylinder with a $2 \mathrm{~mm}$ diameter was used as a counter substrate. For in situ observation of contact formation and detachment, monochromatic illumination of $623 \mathrm{~nm}$, a tubular optic (12X UltraZoom, Navitar Inc., New York, NY, USA), and a camera (DMK 33UX252, ImagingSource, Bremen, Germany) were used. For illustration of long-term attachment behavior, we used interference reflection microscopy (IRM) with a wavelength of $436 \mathrm{~nm}$ and illuminating numerical aperture (INA) of 0.79 . In adhesion tests, the probe was brought into contact with the microstructure at a constant rate of $10 \mu \mathrm{m} \mathrm{s}^{-1}$. Compressive preloads ranged from -2 to $-10 \mathrm{mN}$. The time in contact was $5 \mathrm{~s}$ before the substrate was retracted at a constant velocity of $10 \mu \mathrm{m} \mathrm{s}^{-1}$ until detachment. The maximum tensile force was defined as pull-off force. Each test was performed three times, and mean values were reported. All tests were performed at $21{ }^{\circ} \mathrm{C}$ and a relative humidity of $50 \pm 10 \%$. For the underwater tests, the measurement procedure was identical to that in air, but the microstructures were immersed in a droplet (approximately $50 \mu \mathrm{L}$ ) of distilled water. To ensure complete wetting and avoid entrapped air, the wet specimen was degassed prior to the test at $50 \mathrm{mbar}$ for $\sim 3$ to $5 \mathrm{~min}$ at room temperature. For repeated measurements, the substrate was kept immersed in water.

\section{RESULTS AND DISCUSSION}

3.1. Adhesion Performance in Air of MushroomShaped and $30^{\circ}$ Cupped Microstructures. Cupped microstructures were successfully fabricated from polyurethane by replica molding as illustrated in Figure 1a. The diameter and height of the stalks for all designs were 70 and $100 \mu \mathrm{m}$, respectively. A cup angle of $30^{\circ}$ was initially selected (denoted as $30^{\circ} \mathrm{CM}$, see also Figure $1 \mathrm{~b}$ ). The tip diameter for both the mushroom-shaped (MSM) and the cupped microstructures in their undeformed state was $100 \mu \mathrm{m}$. The thickness of the caps was $\sim 5 \mu \mathrm{m}$ for both structures.

Adhesion of the MSM and $30^{\circ} \mathrm{CM}$ was first measured in air (Figure 2). MSMs showed pull-off forces of $6.7 \pm 0.2 \mathrm{mN}$, corresponding to pull-off stresses of $0.85 \pm 0.03 \mathrm{MPa}$ (mean \pm sd; Figure 2a). It should be noted that the adhesion strengths reported here are calculated on the basis of the real contact area of a single microstructure. For macroscopic arrays, which then consist of many microstructures, the nominal adhesive strength will depend on the areal density of the microstructures and the load distribution across the array. ${ }^{29}$ In the course of $5 \mathrm{~s}$ of preloading (with constant displacement), the compressive force slightly decreased from -3 to $-2.5 \mathrm{mN}$. This force relaxation is related to the viscoelastic relaxation of the polyurethane with a loss factor of 0.12 at $1 \mathrm{~Hz}$ and room temperature. The adhesion-for perfect alignment-was insensitive to preload forces as the contact area between the microstructure and the 

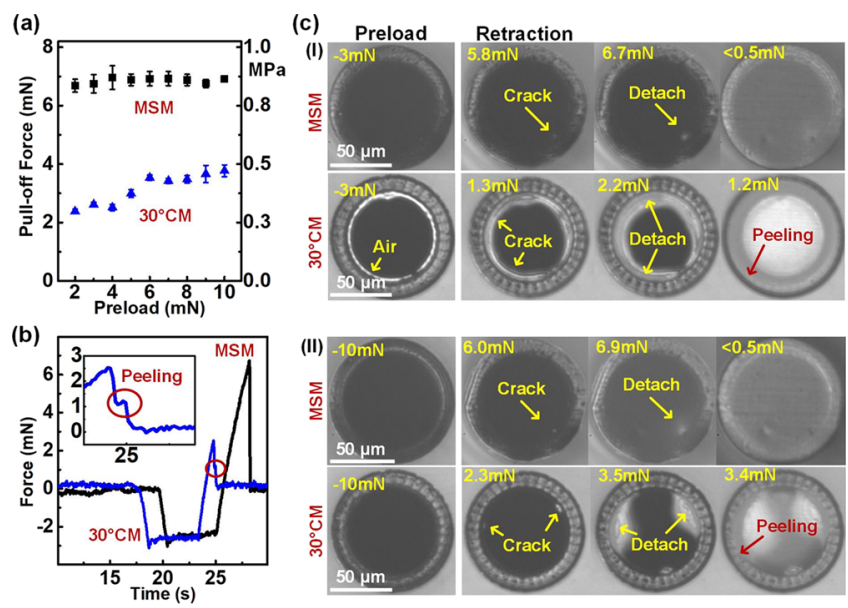

Figure 2. Adhesion tests in air. (a) Pull-off forces for different preloads in mushroom-shaped (MSM) and $30^{\circ}$ cupped microstructures $\left(30^{\circ} \mathrm{CM}\right)$. (b) Typical force-time curves for the two microstructures. The inset represents the detachment of the $30^{\circ} \mathrm{CM}$. (c) Optical micrographs showing the attachment (left) and the detachment process at different tensile loads for both structures after compressive preloading with (I) -3 and (II) $-10 \mathrm{mN}$. Scale bars: $50 \mu \mathrm{m}$.

substrate was the same for all preloads (as depicted for -3 and $10 \mathrm{mN}$ preload in (I) and (II) in Figure 2c, respectively). This insensitivity was only present below a critical buckling load, as reported earlier, for example, in ref 7. Upon tensile loading, detachment of the MSM occurred at the transition from the stalk to the cap that grew into the center via crack propagation (Figure 2c). In this transition region, a tensile stress concentration probably exists, which leads to adhesive failure, in agreement with predictions by Balijepalli et al. ${ }^{14}$ and Spuskanyuk et al. ${ }^{16}$

By contrast, the pull-off forces in air of the cupped microstructures with the $30^{\circ}$ cap $\left(30^{\circ} \mathrm{CM}\right)$ increased with increasing preload and were consistently lower compared to the MSM. At small preloads (as shown for $-3 \mathrm{mN}$ in (I) in Figure $2 \mathrm{c}$ ), air remained trapped in the region of the transition from the stalk to the cap. This defect acted as a critical nucleus that started to grow at $\sim 2 \mathrm{mN}$ tensile load. Interestingly, detachment of the stalk did not result in an immediate drop of the force to zero but produced a $1 \mathrm{mN}$ transient peak (see inset in Figure $2 \mathrm{~b}$ ). In situ observations (see image (I) in Figure 2c) showed that the cap of the microstructure remained in contact during stalk detachment. Final detachment of the cap occurred via peeling at lower force $(\sim 1.2 \mathrm{mN})$ than the initial pull-off (see inset in Figure $2 \mathrm{~b})$. Note that the radial patterns visible along the perimeter of the contact zone are caused by a slight waviness of the surface on both sides of the cap resulting from the fabrication process (see also (II) in Figure $1 b$ ).

We observed less entrapped air inside the $30^{\circ} \mathrm{CM}$ contact zone with increasing preloads. Complete contact with no visible trapped air was achieved at a compressive preload of $-10 \mathrm{mN}$ ((II) in Figure 2c). However, upon retraction of the contact surface, cracks immediately appeared in the transition region between the stalk and the cap. The build-up of high stresses and their concentration in this region likely contributed to crack formation. Even at the highest preload, we cannot exclude the possibility of entrapped air creating defects smaller than the resolution limit of the optical setup. The pull-off forces of $30^{\circ} \mathrm{CM}$ ranged from 2.5 to $4 \mathrm{mN}$ (depending on the preload), corresponding to $25-50 \%$ of the peak forces observed for the MSM. It should be noted that although the geometry of $30^{\circ} \mathrm{CM}$ resembles a suction cup, the stresses within the contact zone ranged between 0.3 and $0.5 \mathrm{MPa}$, which is 3 to 5 times larger than the limit for a purely suction-based mechanism in air (i.e., atmospheric pressure, $0.1 \mathrm{MPa}){ }^{30}$ We conclude that for both the MSM and $30^{\circ} \mathrm{CM}$, the adhesive forces in air are mainly the result of van der Waals interactions and resistance to crack initiation and propagation, which in turn depends on interfacial stress distribution, stiffness, and the presence of interfacial defects. ${ }^{29,31,32}$

3.2. Comparing Underwater Adhesion Performance between Mushroom-Shaped and $30^{\circ}$ Cupped Microstructures. Adhesion tests were performed in water using both the MSM and $30^{\circ} \mathrm{CM}$ to understand the effect of the cap design on underwater adhesion (Figure 3). Consistent with previous

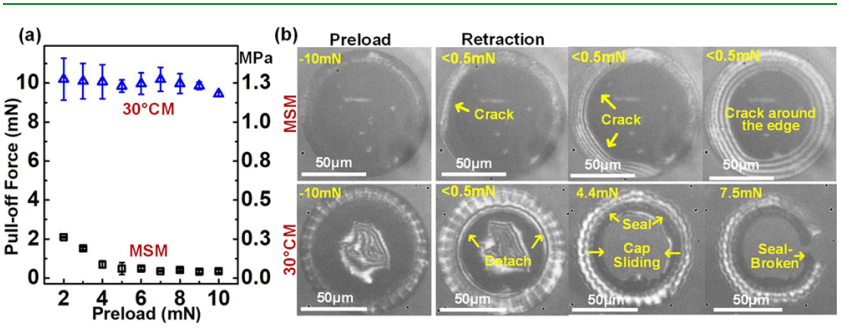

Figure 3. Underwater adhesion tests. (a) Pull-off forces in terms of preloads for mushroom-shaped (MSM) and $30^{\circ}$ cupped microstructures $\left(30^{\circ} \mathrm{CM}\right)$. (b) Optical micrographs showing the attachment (left) and the detachment process at different tensile loads for both structures after compressive preloading of $-10 \mathrm{mN}$. Scale bars: $50 \mu \mathrm{m}$.

studies, ${ }^{18,20}$ we found that the underwater adhesion force of the MSM was significantly lower than in air: between 0.5 and $2 \mathrm{mN}$ in water compared to $7 \mathrm{mN}$ in air. Furthermore, in underwater tests, the MSM detached from the edge with the crack propagating along the rim circumference before central stalk detachment, which is opposite to the behavior in air where cracks propagated from the center to the edge of the contact (Figure 3b). From earlier reports, it is known that surface wettability has a significant impact on adhesion performance in wet environments. ${ }^{17,20}$ For hydrophilic materials, a thermodynamically stable layer of water promotes separation of the solidsolid contact, and van der Waals interactions and adhesion are significantly reduced. ${ }^{19}$ In our studies, the glass probe and the polyurethane used to fabricate the microstructures were hydrophilic with a static contact angle of $\sim 26$ and $83^{\circ}$, respectively (as shown in Figure S2 in the Supporting Information).

In strong contrast to the MSM, $30^{\circ} \mathrm{CM}$ showed a remarkable improvement in adhesion when tested underwater: Peak pull-off forces were around $10 \mathrm{mN}$ (corresponding to a pull-off stress of 1.3 MPa), more than 2-fold higher than in air. Interestingly, this improvement was largely independent of preload, in contrast to the behavior in air where higher preloads led to increased pull-off forces. Most strikingly, the peak pull-off force of $30^{\circ} \mathrm{CM}$ exceeded the performance of the MSM by up to 20 -fold (depending on the preload). Since the test conditions and materials were kept constant between the MSM and $30^{\circ} \mathrm{CM}$, this large improvement in underwater adhesion is based on the cap geometry of the $30^{\circ} \mathrm{CM}$.

Figure 4 shows a schematic representation of the potential mechanisms underlying the performance enhancement of $30^{\circ} \mathrm{CM}$. During attachment, the rim of the cupped microstructure first makes contact with the substrate and is elastically deformed until the set preload is reached, as shown in (I) in 


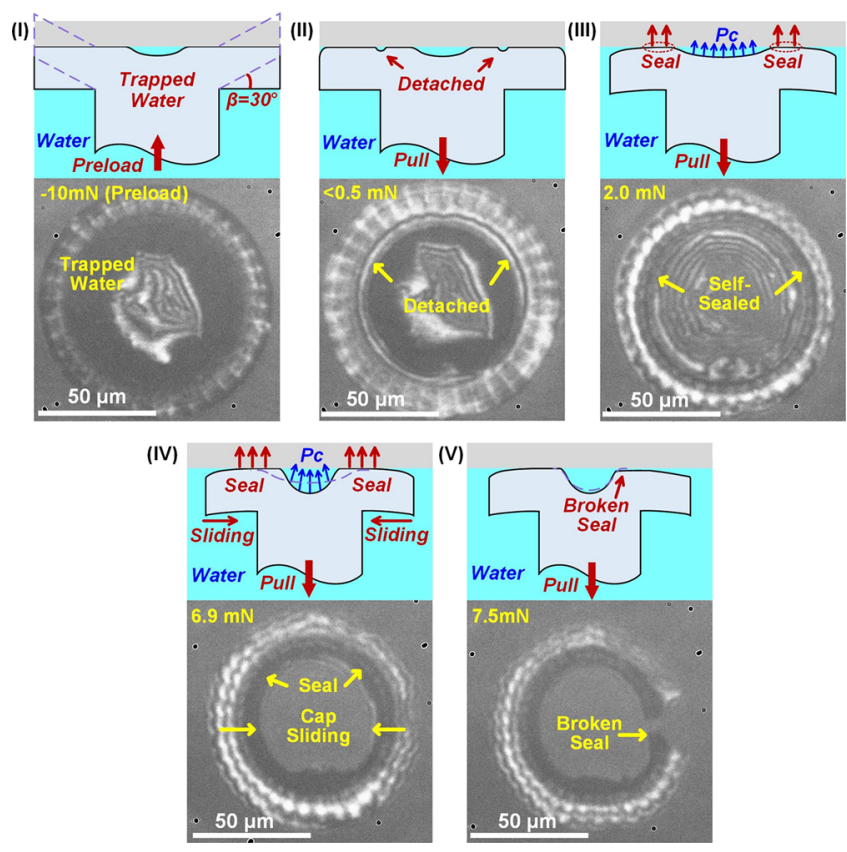

Figure 4. Image sequence illustrating the underwater detachment of the $30^{\circ}$ cupped microstructure. Optical micrographs show the contact zone, while illustrations represent side views to depict underlying mechanisms: (I) initial contact of the cupped microstructure upon compressive preloading of the cup (dashed lines for the undeformed state). (II) The structure partially detaches while water starts to flow into the contact. (III) The hydrostatic pressure, $p_{\mathcal{c}}$, of the entrapped water underneath the contact decreases, leading to seal formation. (IV) The sealed contact shrinks by inward sliding of the seal. (V) The seal breaks by inward collapse of the cup just before detachment. Scale bars: $50 \mu \mathrm{m}$.

Figure 4. During preloading, water is squeezed out from underneath the cup until the stalk makes contact with the substrate. Consistently, some residual water remains trapped within the contact zone (see optical micrographs in (I) in Figure 4 or Figure 3b). During force relaxation (II), water rapidly flows into the contact, suggesting that the contact is not well sealed at this point. In the tensile loading stage (III and IV), however, a better seal (see dark gray ring in the respective optical micrographs) between the cap and the substrate is formed, not at its outermost rim but further inward, encompassing the entrapped water (III). Perhaps counter-intuitively, the outermost rim is first detached from the surface and therefore contributes minimally to the sealing of the contact area. The more internal seal seems to form when the flexible walls of the cup are pulled into close contact with the substrate as a result of the low hydrostatic pressure underneath the cup. Interestingly, a better seal with less leakage is generated at higher tensile loads (IV); therefore, $30^{\circ} \mathrm{CM}$ is a self-sealing structure. It should be noted that even this improved seal is not completely leak-proof since we observed influx of water at all stages of the retraction, as shown below in more detail. The final detachment of $30^{\circ} \mathrm{CM}$ underwater appears to be two-phasic (V): First, the stalk gradually detaches by crack nucleation from defects or entrapped water and subsequent crack propagation (II and III). Once the stalk detaches, the outer rim edge and internal seal begin to contract by sliding inward (observed for tensile loads larger than $5 \mathrm{mN}$, see IV). The rim diameter was observed to decrease by approximately $30 \%$ from fully preloaded to immediately prior to detachment, as shown in Figure S3 in the
Supporting Information. This contact shrinkage is likely a consequence of the lowered hydrostatic pressure and the incompressibility of the entrapped water under the cup: a vertical pull on the cavity under the cup induces a centripetal (inward) pull on the rim. Note that a lateral contact slippage could be eased by the presence of water in the interface possibly acting as a lubricant. This slippage was found to be less pronounced in air where the contact area remained almost constant until detachment (Figure S3 in the Supporting Information). The final detachment occurs by a segment of the rim collapsing inward (V), leading to a large water influx, equilibration of the pressure, and complete detachment of the microstructure (see Video S1).

It should be noted that no cavitation, that is, pore formation inside the water volume, was observed for any of the underwater tests with cupped microstructures where peak pull-off strengths exceeded 1 atm. Many examples of water under negative pressure can be found in nature: Water columns within xylem vessels of trees can be under tension of up to $1.8 \mathrm{MPa}{ }^{33}$ while water inside the cavity of octopus and squid suckers can withstand pressure differences of up to $0.8 \mathrm{MPa}^{34,35}$ In fact, even at the extreme pull-off strengths of $1.3 \mathrm{MPa}$ or $\sim 13 \mathrm{~atm}$, there was no cavitation observed in our experiments, and detachment occurred by the mechanisms stated above. Under these conditions, water in the contact zone is subjected to pressures well below $0 \mathrm{~atm}$; hence, the water is in a metastable state. ${ }^{36}$ The levels of negative pressure observed here strongly exceed those found in studies using larger-scale probe-tack adhesion tests. ${ }^{37}$ It is likely that the smaller contact size of our microstructures results in thinner fluid films in the contact zone and thereby limits cavitation by preventing gas bubbles from reaching the critical size at which they would grow explosively. ${ }^{37}$ As our cupped microstructures did not detach via cavitation, it is likely that we did not reach the maximum pull-off forces possible with this design.

To further investigate the self-sealing behavior of the microstructures, hold time tests at different tensile loads were performed in water (Figure 5). For this experiment, $30^{\circ} \mathrm{CM}$ was brought into contact with the substrate at $-10 \mathrm{mN}$ preload. The substrate was then retracted to a set tensile load $F_{\Delta}$ where the position (displacement) was fixed and the time to detachment was recorded. Figure 5a shows the force-time curve. The applied tensile force of $2.2 \mathrm{mN}$ slowly decreased over time until the seal broke and detachment occurred after approximately 6 $\mathrm{min}$. Figure $5 \mathrm{~b}$ summarizes the times the microstructures stayed in contact with the substrate for various tensile forces $F_{\Delta}$. The time to detachment increased from $\sim 6 \mathrm{~min}$ for $2.2 \mathrm{mN}$ to $\sim 22$ $\min$ for $3.2 \mathrm{mN}$. This delayed detachment is associated with a lower leakage rate due to better adaptation of the microstructure to the substrate. This characteristic proves the self-sealing mechanism related to the compressive forces at the interface induced by the pressure difference between inside and outside the cavity. For larger forces $(>4.2 \mathrm{mN})$, the time to detachment decreased to less than $1 \mathrm{~min}$ for $7.6 \mathrm{mN}$. Now, the high leakage rate increased again, which was most probably caused by high elastic distortions due to excessive tensile loads.

For a given seal, the flow rate is proportional to the pressure gradient. Hence, the quality of the seal can be estimated from the product of time and applied tensile force (red curve in Figure $5 b$ ). At forces $F_{\Delta}<4.2 \mathrm{mN}$, the quality of the seal increased with the strength of the pull, demonstrating the self-sealing properties of the cupped microstructure. This effect may be based on the increased pressure difference between inside and outside the 

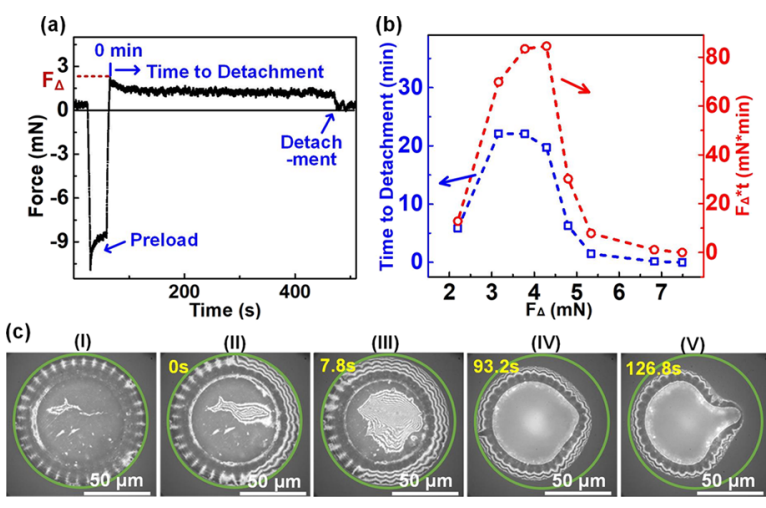

Figure 5. Hold time experiments for the $30^{\circ}$ cupped microstructure. (a) Force versus time for a set tensile load of $2.2 \mathrm{mN}$. (b) Time to detachment as a function of applied tensile forces, $F_{\Delta}$ (blue curve). The red curve depicts the product of force and detachment time, $F_{\Delta} \times t$, as a function of the applied tensile force. (c) Image sequence illustrating seal formation, leakage, and failure by sliding and inward collapse for an initially set tensile load of $5 \mathrm{mN}$ (I). Green circular outline marks the full contact area at preload. (II, III) The increase in water volume (evident in the increasing number of interference fringes and area occupied by them) at the center demonstrates leakage flow. (IV) The growing water volume in the center completely detached the stalk. (V) Immediately prior to detachment, the upper right section of the rim collapsed inward. Scale bar: $50 \mu \mathrm{m}$.

contact zone, which "pulls" the outer rim into even closer contact, thereby improving the seal. At forces $F_{\Delta}>4.2 \mathrm{mN}$, the quality of the seal decreased, likely as a result of the increasing strain on the cup. We conclude that the cupped microstructures exhibit self-sealing properties, and the quality of their seal critically depends on the applied tensile load, which in this case was maximal at $40-50 \%$ of the peak pull-off force.

The image sequence of the detachment after $-5 \mathrm{mN}$ preloading is shown in Figure 5c. Even after the internal seal was formed at $5 \mathrm{mN}$ tensile load (II), the increasing number of interference fringes and area of the volume underneath the cup indicated leakage and water flowing into the contact over time (III and IV). After $93 \mathrm{~s}$, the growing water volume in the center completely detached the stalk, yet the internal seal remained intact $(\mathrm{V})$. During the test, the rim continuously slid inward at the right side of the cup, which immediately prior to detachment induced an inward collapse of the remaining rim (VI).

The leakage rate was not constant during the retraction phase but showed a stepwise behavior: We observed a relatively steady increase in internal water volume punctuated by large influxes of water (see Video S1). The leakage rates and the detailed mechanisms underlying these stochastic influxes will be the subject of further investigations.

3.3. Optimizing the Design of Cupped Microstructures. Our results on the $30^{\circ}$ cupped microstructure $\left(30^{\circ} \mathrm{CM}\right)$ have shown that the cupped geometry impacts the adhesive performance under wet and dry conditions. The mushroomshaped microstructure (MSM) performed better than $30^{\circ} \mathrm{CM}$ in air, but in wet conditions, $30^{\circ} \mathrm{CM}$ drastically outperformed the MSM. Since the $30^{\circ}$ cup angle was chosen arbitrarily, we investigated the effect of varying cup angles on adhesive performance, with the goal of finding an optimum angle for strong adhesion in both air and water. Microstructures with 15 and $45^{\circ}$ cups $\left(15^{\circ} \mathrm{CM}\right.$ and $45^{\circ} \mathrm{CM}$, respectively) were fabricated and tested in comparison to the results presented above (Figure 6a). In air, $15^{\circ} \mathrm{CM}$ achieved pull-off forces 2.5 times higher than
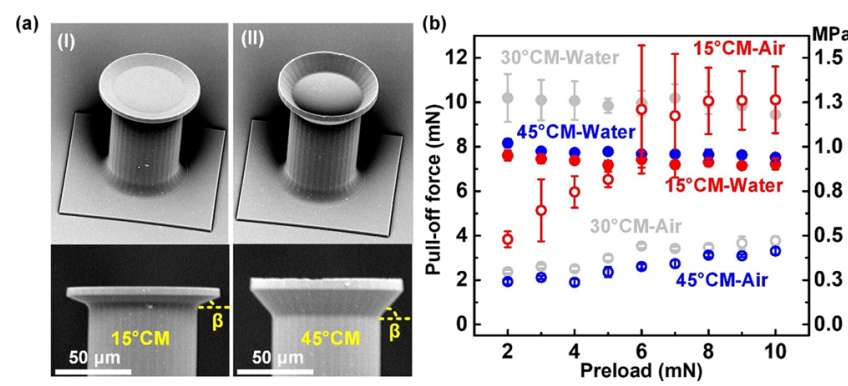

(c) $15^{\circ} \mathrm{CM}-\mathrm{Air}$

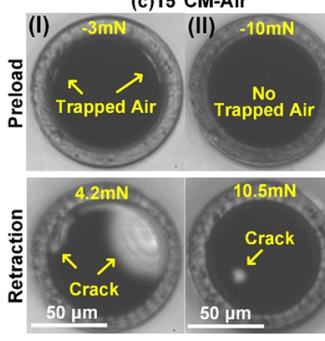

(d) $45^{\circ} \mathrm{CM}-\mathrm{Air}$

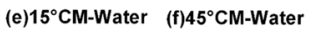

Figure 6. Adhesion tests for 15,30 , and $45^{\circ}$ cupped microstructures. (a) Electron micrographs showing top and side views of $15^{\circ} \mathrm{CM}$ and $45^{\circ} \mathrm{CM}$ (I and II, respectively). (b) Pull-off forces in terms of preload for $15^{\circ} \mathrm{CM}$ (red) and $45^{\circ} \mathrm{CM}$ (blue) in comparison to $30^{\circ} \mathrm{CM}$ (gray) tested in air (unfilled symbols) and underwater (filled symbols). (c-f) Optical micrographs showing the attachment upon preload and the detachment process of the (c) $15^{\circ} \mathrm{CM}$ at (I) $-3 \mathrm{mN}$ and (II) $-10 \mathrm{mN}$ preload and (d) $45^{\circ} \mathrm{CM}$ tested in air and tested underwater (e) $15^{\circ} \mathrm{CM}$ and (f) $45^{\circ} \mathrm{CM}$. Scale bars: $50 \mu \mathrm{m}$.

$30^{\circ} \mathrm{CM}(\sim 10 \mathrm{mN}$ vs $\sim 4 \mathrm{mN}$, see Figure $6 \mathrm{~b}), 3$ times higher than $45^{\circ} \mathrm{CM}(\sim 10 \mathrm{mN}$ vs $\sim 3 \mathrm{mN})$, and even exceeding the MSM $(\sim 10 \mathrm{mN}$ vs $7 \mathrm{mN})$. This result confirms that the cup angle can be optimized to increase adhesion in dry conditions. A similar result was recently reported by Fischer et al. ${ }^{11}$ Cupped microstructures attach gradually to the substrate by bending and storing elastic energy (whereas mushroom structures with flat ends attach in one step). Thus, the cup makes contact with the substrate first with its outer rim before complete contact is established under compressive loading. This explains why the adhesive performance of these structures is sensitive to the preload conditions: as shown in Figure $6 \mathrm{c}$ for dry adhesion tests with $15^{\circ} \mathrm{CM}$, air pockets remained in the transition region from the stalk to the cap at small preloads of $-3 \mathrm{mN}$. The trapped air acted as a pre-existing crack and initiated separation during retraction. At compressive preloads greater than $-6 \mathrm{mN}$, however, no trapped air was observed, and the central stalk came into full contact. Detachment occurred from the inner region of the stalk at higher tensile loads (Figure 6c). This contrasts with $45^{\circ} \mathrm{CM}$ where trapped air remained within the cavity even at a large preload of $-10 \mathrm{mN}$ (Figure 6d). This entrapment always led to crack propagation, explaining the poorer performance of $45^{\circ} \mathrm{CM}$ compared to $15^{\circ} \mathrm{CM}$.

Our findings suggest that $15^{\circ} \mathrm{CM}$ outperforms mushroomshaped microstructures in air by reducing the magnitude of interfacial tensile stresses near the edge of the rim due to the compression of the cap during loading. Such a reduction has previously been shown for the MSM to lead to higher adhesion. ${ }^{11,14,38}$ This advantage, however, comes with a tradeoff for the cupped structure, since bending upon preload results in stored elastic strain energy that counteracts adhesion. This is the likely the reason why the pull-off force decreased with increasing tilt angles $(10 \mathrm{mN} \rightarrow 4 \mathrm{mN} \rightarrow 3 \mathrm{mN}$, for cup angles increasing from $15^{\circ} \rightarrow 30^{\circ} \rightarrow 45^{\circ}$ ). It is also possible that the 
peeling of the cup from the center to the edge induces a shear stress component that may increase adhesion. ${ }^{39,40}$

For underwater tests, both $15^{\circ} \mathrm{CM}$ and $45^{\circ} \mathrm{CM}$ showed strong adhesion (in the range of 7 to $8 \mathrm{mN}$ ) for all given preloads (Figure 6b). The detachment process was similar to that described above for $30^{\circ} \mathrm{CM}$ : Upon retraction, water leaked slowly through the seal into the central cavity throughout the detachment phase. During this process, the seal slowly slid inward. Full detachment was preceded by inward collapse of the cup walls (Figure 6e,f). We measured similar pull-off forces underwater, despite the variation in geometry, preloads, and volume of water trapped within the cavity. However, we observed in all cases that water flowed into the contact area when the preload was released. Thus, the amount of water entering the contact zone depends on the hydrodynamic conditions, which are controlled by the pressure gradient. As the retraction velocity determines the pressure produced (probably more than the difference in cup angle), it controls the time until a seal is formed. Retraction velocity and wettability was kept constant in our study; hence, no variation in the underwater performance was observed.

Since we found self-sealing behavior underwater with $30^{\circ} \mathrm{CM}$, we tested whether this effect also applied to $15^{\circ} \mathrm{CM}$. At low tensile loads ( 2 to $2.5 \mathrm{mN}$ ), the seal was not well established, resulting in water inflow and detachment within a few minutes (Figure 7a). At higher tensile loads (between 30 and $60 \%$ of the

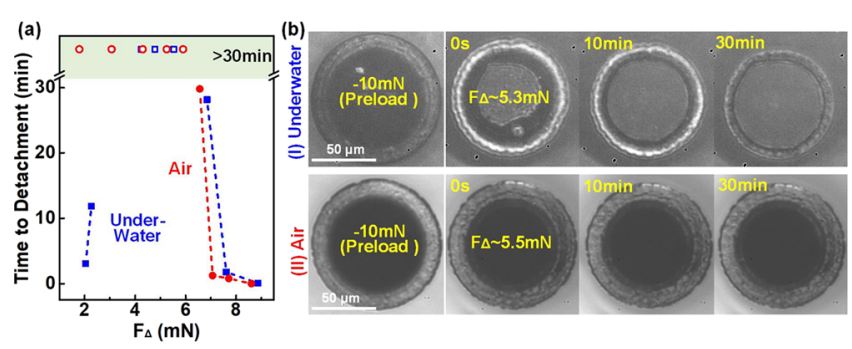

Figure 7 . Hold time experiments of the $15^{\circ}$ cupped microstructure in air and water. (a) Time to detachment as a function of applied tensile force, $F_{\Delta}$, tested in air (red circles) and underwater (blue squares). All tests were stopped after $30 \mathrm{~min}$. Tests where the structure remained in contact for $>30 \mathrm{~min}$ are shown as open symbols. (b) Optical micrographs showing the preload (left) and the detachment process over time (I) underwater and (II) in air. The applied tensile force was 5.3 and $5.5 \mathrm{mN}$. Scale bars: $50 \mu \mathrm{m}$.

pull-off force), however, the structures stayed in contact for at least $30 \mathrm{~min}$, at which point the experiments were stopped. At even higher applied forces, the detachment occurred within a few minutes for $7 \mathrm{mN}$ and within seconds for $8.5 \mathrm{mN}$. As shown in (I) in Figure $7 \mathrm{~b}$ (for an applied tensile load of $5.3 \mathrm{mN}$ ), the location of the sealing rim moved outward, while water leaked slowly and continuously into the contact zone. In contrast to the situation underwater, the tests in air did not show any changes in the contact geometry up to the start of crack propagation and final detachment (II). All structures stayed in contact with the substrate for at least $30 \mathrm{~min}$ when steady forces below $6 \mathrm{mN}$ were applied.

\section{CONCLUSIONS}

A unique property of the cupped microstructures developed in this study is that they show strong adhesion both in air and underwater, thereby outperforming flat mushroom-shaped microstructures. By reducing the cup angle to $15^{\circ}$, we successfully improved their adhesive strength in air while retaining their underwater performance. The following conclusions can be drawn:

- Mushroom-shaped fibers produced high adhesive strength only in air (up to $0.9 \mathrm{MPa}$ ) but not underwater $(\sim 0.05 \mathrm{MPa})$. In contrast, $30^{\circ} \mathrm{CM}$ showed slightly reduced adhesion in air $(0.3-0.5 \mathrm{MPa})$ but much stronger adhesion underwater (exceeding 1.3 MPa). For cup angles of $15^{\circ}$, adhesion in air even exceeded that of mushroom-shaped structures (around 1.3 MPa), while underwater adhesion was still very strong $(1 \mathrm{MPa})$ (see Figure 8).

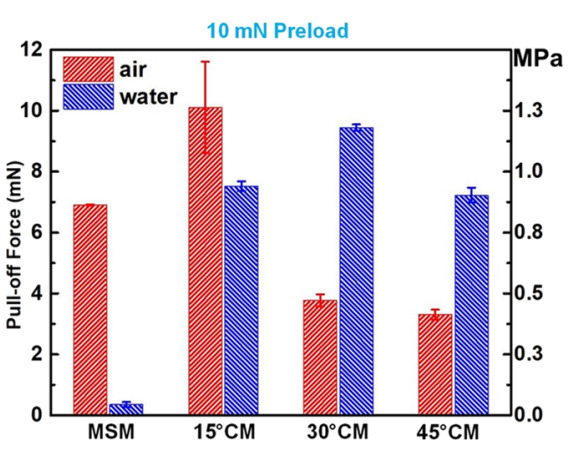

Figure 8. Air and underwater adhesion of all structures tested in this study upon $10 \mathrm{mN}$ compressive preload.

- Despite similar adhesive strength in air and underwater, the cupped microstructures showed different detachment processes in the two media. In air, the cap separated by rapid crack propagation, whereas underwater, the cap separated gradually via water leaking through the seal into the contact zone until the rim collapsed inward and detached. While the slight loss of adhesion of $30^{\circ} \mathrm{CM}$ in air is probably explained by air trapped in the contact zone that initiates the formation of cracks and leads to faster detachment, the gain in adhesion for $15^{\circ} \mathrm{CM}$ may be based on a more uniform interfacial stress distribution, leading to higher pull-off forces.

- The dramatic increase of underwater adhesion achieved by cupped microstructures is based on the establishment of a tight seal that almost completely blocks the flow of water into the contact zone. Interestingly, our results provide clear evidence that the cupped microstructures possess self-sealing properties: higher tensile forces result in a better seal and improved close contact, resulting in longer attachment times compared to smaller tensile forces.

In summary, cupped microstructures provide an effective solution for high adhesion in both wet and dry conditions, which is superior to previous designs of fibrillar adhesives. As variable wetness is common in many environments where adhesive applications are needed, our study may guide the development of adhesives that are insensitive to such changing conditions.

\section{ASSOCIATED CONTENT}

\section{S Supporting Information}

The Supporting Information is available free of charge on the ACS Publications website at DOI: 10.1021/acsami.9b07969.

Image and schematic illustration of the custom-made test apparatus; contact angle measurements; reduction of 
contact area at pull-off in comparison to the contact area at preload in air and underwater (PDF)

Underwater attachment and detachment of the $30^{\circ} \mathrm{CM}$ (AVI)

\section{AUTHOR INFORMATION}

\section{Corresponding Authors}

*E-mail: wf222@cam.ac.uk (W.F.).

*E-mail: rene.hensel@leibniz-inm.de (R.H.).

ORCID ${ }^{\circ}$

René Hensel: 0000-0002-9623-2118

Notes

The authors declare no competing financial interest.

\section{ACKNOWLEDGMENTS}

The authors would like to greatly thank Joachim Blau and Simon Chen for their help in building the various experimental setups. The research leading to these results was partially funded by the European Research Council under the European Union's Seventh Framework Programme (FP/2007-2013)/ERC Grant Agreement no. 340929 and through the European Union's Horizon 2020 Research and Innovation Programme under the Marie Skłodowska-Curie grant agreement no 642861.

\section{REFERENCES}

(1) Estrada, M. A.; Hockman, B.; Bylard, A.; Hawkes, E. W.; Cutkosky, M. R.; Pavone, M. Free-Flyer Acquisition of Spinning Objects with Gecko-Inspired Adhesives. In Proceedings - IEEE International Conference on Robotics and Automation; 2016; Vol. 2016-, pp 4907-4913.

(2) Li, Y.; Ahmed, A.; Sameoto, D.; Menon, C. Abigaille II: Toward the Development of a Spider-Inspired Climbing Robot. Robotica 2012, 30, 79-89.

(3) Jeong, H. E.; Lee, J.-K.; Kim, H. N.; Moon, S. H.; Suh, K. Y. A Nontransferring Dry Adhesive with Hierarchical Polymer Nanohairs. Proc. Natl. Acad. Sci. 2009, 106, 5639-5644.

(4) Zhou, M.; Tian, Y.; Sameoto, D.; Zhang, X.; Meng, Y.; Wen, S. Controllable Interfacial Adhesion Applied to Transfer Light and Fragile Objects by Using Gecko Inspired Mushroom-Shaped Pillar Surface. ACS Appl. Mater. Interfaces 2013, 5, 10137-10144.

(5) Purtov, J.; Frensemeier, M.; Kroner, E. Switchable Adhesion in Vacuum Using Bio-Inspired Dry Adhesives. ACS Appl. Mater. Interfaces 2015, 7, 24127-24135.

(6) Song, S.; Drotlef, D.-M.; Majidi, C.; Sitti, M. Controllable Load Sharing for Soft Adhesive Interfaces on Three-Dimensional Surfaces. Proc. Natl. Acad. Sci. 2017, 114, E4344-E4353.

(7) Tinnemann, V.; Arzt, E.; Hensel, R. Switchable Double-Sided Micropatterned Adhesives for Selective Fixation and Detachment. J. Mech. Phys. Solids 2019, 123, 20-27.

(8) Hensel, R.; Moh, K.; Arzt, E. Engineering Micropatterned Dry Adhesives: From Contact Theory to Handling Applications. Adv. Funct. Mater. 2018, 28, 1800865.

(9) Kamperman, M.; Kroner, E.; del Campo, A.; McMeeking, R. M.; Arzt, E. Functional Adhesive Surfaces with "Gecko" Effect: The Concept of Contact Splitting. Adv. Eng. Mater. 2010, 12, 335-348.

(10) Del Campo, A.; Greiner, C.; Arzt, E. Contact Shape Controls Adhesion of Bioinspired Fibrillar Surfaces. Langmuir 2007, 23, 1023510243.

(11) Fischer, S. C. L.; Groß, K.; Torrents Abad, O.; Becker, M. M.; Park, E.; Hensel, R.; Arzt, E. Funnel-Shaped Microstructures for Strong Reversible Adhesion. Adv. Mater. Interfaces 2017, 4, 1700292.

(12) Fischer, S. C. L.; Arzt, E.; Hensel, R. Composite Pillars with a Tunable Interface for Adhesion to Rough Substrates. ACS Appl. Mater. Interfaces 2017, 9, 1036-1044.

(13) Minsky, H. K.; Turner, K. T. Achieving Enhanced and Tunable Adhesion via Composite Posts. Appl. Phys. Lett. 2015, 106, 201604.
(14) Balijepalli, R. G.; Begley, M. R.; Fleck, N. A.; McMeeking, R. M.; Arzt, E. Numerical Simulation of the Edge Stress Singularity and the Adhesion Strength for Compliant Mushroom Fibrils Adhered to Rigid Substrates. Int. J. Solids Struct. 2016, 85-86, 160-171.

(15) Heepe, L.; Gorb, S. N. Biologically Inspired Mushroom-Shaped Adhesive Microstructures. Annu. Rev. Mater. Res. 2014, 44, 173-203.

(16) Spuskanyuk, A. V.; McMeeking, R. M.; Deshpande, V. S.; Arzt, E. The Effect of Shape on the Adhesion of Fibrillar Surfaces. Acta Biomater. 2008, 4, 1669-1676.

(17) Stark, A. Y.; Badge, I.; Wucinich, N. A.; Sullivan, T. W.; Niewiarowski, P. H.; Dhinojwala, A. Surface Wettability Plays a Significant Role in Gecko Adhesion Underwater. Proc. Natl. Acad. Sci. U. S. A. 2013, 110, 6340-6345.

(18) Heepe, L.; Kovalev, A. E.; Gorb, S. N. Direct Observation of Microcavitation in Underwater Adhesion of Mushroom-Shaped Adhesive Microstructure. Beilstein J. Nanotechnol. 2014, 5, 903-909.

(19) Cadirov, N.; Booth, J. A.; Turner, K. L.; Israelachvili, J. N. Influence of Humidity on Grip and Release Adhesion Mechanisms for Gecko-Inspired Microfibrillar Surfaces. ACS Appl. Mater. Interfaces 2017, 9, 14497-14505.

(20) Soltannia, B.; Sameoto, D. Strong, Reversible Underwater Adhesion via Gecko-Inspired Hydrophobic Fibers. ACS Appl. Mater. Interfaces 2014, 6, 21995-22003.

(21) Israelachvili, J. N. Intermolecular and Surface Forces: Third Edition; Elsevier: 2011. DOI: 10.1016/C2011-0-05119-0.

(22) Stark, A. Y.; McClung, B.; Niewiarowski, P. H.; Dhinojwala, A. Reduction of Water Surface Tension Significantly Impacts Gecko Adhesion Underwater. Integr. Comp. Biol. 2014, 54, 1026-1033.

(23) Lee, H.; Lee, B. P.; Messersmith, P. B. A Reversible Wet/Dry Adhesive Inspired by Mussels and Geckos. Nature 2007, 448, 338-341.

(24) Ma, Y.; Ma, S.; Wu, Y.; Pei, X.; Gorb, S. N.; Wang, Z.; Liu, W.; Zhou, F. Remote Control over Underwater Dynamic Attachment/ Detachment and Locomotion. Adv. Mater. 2018, 30, 1801595.

(25) Zhao, Y.; Wu, Y.; Wang, L.; Zhang, M.; Chen, X.; Liu, M.; Fan, J.; Liu, J.; Zhou, F.; Wang, Z. Bio-Inspired Reversible Underwater Adhesive. Nat. Commun. 2017, 8, 2218.

(26) Rao, P.; Sun, T. L.; Chen, L.; Takahashi, R.; Shinohara, G.; Guo, H.; King, D. R.; Kurokawa, T.; Gong, J. P. Tough Hydrogels with Fast, Strong, and Reversible Underwater Adhesion Based on a Multiscale Design. Adv. Mater. 2018, 30, 1801884.

(27) Baik, S.; Kim, D. W.; Park, Y.; Lee, T.-J.; Bhang, S. H.; Pang, C. A Wet-Tolerant Adhesive Patch Inspired by Protuberances in Suction Cups of Octopi. Nature 2017, 546, 396-400.

(28) Purtov, J.; Verch, A.; Rogin, P.; Hensel, R. Improved Development Procedure to Enhance the Stability of Microstructures Created by Two-Photon Polymerization. Microelectron. Eng. 2018, 4550.

(29) Tinnemann, V.; Hernández, L.; Fischer, S. C. L.; Arzt, E.; Bennewitz, R.; Hensel, R. In Situ Observation Reveals Local Detachment Mechanisms and Suction Effects in Micropatterned Adhesives. Adv. Funct. Mater. 2019, 29, 1807713.

(30) Denny, M. Biology and the Mechanics of the Wave-Swept Environment; Princeton University Press: 2014, Vol. 917.

(31) Bin Khaled, W.; Sameoto, D. Anisotropic Dry Adhesive via Cap Defects. Bioinspir. Biomim. 2013, 8 ().

(32) Heepe, L.; Kovalev, A. E.; Filippov, A. E.; Gorb, S. N. Adhesion Failure at 180000 Frames per Second: Direct Observation of the Detachment Process of a Mushroom-Shaped Adhesive. Phys. Rev. Lett. 2013, 111, 104301.

(33) Koch, G. W.; Stillet, S. C.; Jennings, G. M.; Davis, S. D. The Limits to Tree Height. Nature 2004, 428, 851-854.

(34) Smith, A. M. Negative Pressure Generated by Octopus Suckers: A Study of the Tensile Strength of Water in Nature. J. Exp. Biol. 1991, 157, 257-271.

(35) Smith, A. Cephalopod Sucker Design and the Physical Limits to Negative Pressure. J. Exp. Biol. 1996, 199, 949-958.

(36) Caupin, F.; Herbert, E. Cavitation in water: a review Cavitation dans l'eau : revue. C. R. Phys. 2006, 7, 1000-1017. 
(37) Poivet, S.; Nallet, F.; Gay, C.; Teisseire, J.; Fabre, P. Force Response of a Viscous Liquid in a Probe-Tack Geometry: Fingering versus Cavitation. Eur. Phys. J. E: Soft Matter Biol. Phys. 2004, 97-116.

(38) Balijepalli, R. G.; Fischer, S. C. L.; Hensel, R.; McMeeking, R. M.; Arzt, E. Numerical Study of Adhesion Enhancement by Composite Fibrils with Soft Tip Layers. J. Mech. Phys. Solids 2017, 99, 357-378.

(39) Santos, D.; Spenko, M.; Parness, A.; Kim, S.; Cutkosky, M. Directional Adhesion for Climbing: Theoretical and Practical Considerations. J. Adhes. Sci. Technol. 2007, 21, 1317-1341.

(40) Zhou, M.; Pesika, N.; Zeng, H.; Tian, Y.; Israelachvili, J. Recent Advances in Gecko Adhesion and Friction Mechanisms and Development of Gecko-Inspired Dry Adhesive Surfaces. Friction 2013, 1, 114129. 